\title{
Mamíferos y Proyecto Hidroeléctrico San Rafael, sureste de Costa Rica
}

\author{
LUIS Diego ARIAS-CAMPOS \\ Escuela de la Tierra, Coordinador Programa de Biología Tropical, Longo Mai, Costa Rica; \\ E-mail: diegoac33@hotmail.com
}

Recibido: 27 setiembre 2017

Aceptado: 22 octubre 2017

\begin{abstract}
RESUMEN
En este trabajo se muestra las especies de mamíferos no voladores que habitan la cuenca del río Unión, de forma comparativa con los presentados por el Estudio de Impacto Ambiental (EsIA) del Proyecto Hidroeléctrico San Rafael. Los datos obtenidos son producto de observaciones personales de junio de 2014 a junio 2015, y reportes de lugareños. Se incluyen 29 especies clasificadas en 16 familias, mientras que el EsIA reportó ocho. El hábito más común es frugivoría. La familia con más especies fue Didelphidae. Cuatro especies están bajo alguna categoría de amenaza. Tres poseen poblaciones reducidas o amenazadas, y tres se encuentran en peligro de extinción, datos no presentados en el EsIA en la mayoría de los casos. Los ecosistemas ribereños representan un importante hábitat para las especies en general, cuyos impactos por los proyectos hidroeléctricos no logran ser medidos a través de los EsIA.
\end{abstract}

Palabras clave: río; bosque ribereño; estado de conservación; fragmentación.

\section{ABSTRACT \\ MAMÍFEROS Y PROYECTO HIDROELÉCTRICO SAN RAFAEL, SURESTE DE COSTA RICA}

This work shows the species of non-flying mammals that inhabit the basin of the Union river, comparing with those presented by the Environmental Impact Study (EIS) of the San Rafael Hydroelectric Project. The data obtained are the product of personal observations from June 2014 to June 2015, and reports of villagers. It includes 29 species classified in 16 families, while the EIS reported eight. The most common habit is frugivory. The family with the most species was Didelphidae. Four species are under some category of threat. Three have small or threatened populations, and three are in danger of extinction, data not presented in the EIS in most cases. Riparian ecosystems represent an important habitat for species in general, whose impacts on hydroelectric projects can not be measured through EIS.

Key words: river; riverine forest; condition; fragmentation.

\section{Introducción}

En Costa Rica se conocen 249 especies de mamíferos; 35 acuáticos y 214 terrestres, de los cuales 114 son voladores (Chiroptera); el de más especies en el país. Las restantes 100 son terrestres (Rodríguez, Ramírez, Villalobos y Sánchez, 2014). Se caracterizan por la presencia de glándulas mamarias, pelo, y la mayoría con reproducción vivípara. Las dietas incluyen plantas, insectos, mamíferos, peces, néctar, carroña, frutos, semillas, raíces, y a veces restos de comida humana (Carrillo y Vaughan, 1993; Mora, 2000; Wainwright, 2002).

Algunas especies han logrado adaptarse en sitios con influencia antrópica (Carrillo y Vaughan, 1993; Monge y Linares, 2010), convirtiéndose en ocasiones perjudiciales para las actividades humanas (Hilje y Monge, 1988). Por el contrario, otras están dirigidas hacia la reducción de poblaciones debido a la pérdida de hábitat, disminución de alimento, cacería, atropellos, etc. (Spinola y Vaughan, 1994; Monge, 1996).

El auge de los proyectos hidroeléctricos en el país responden a una iniciativa de regionalización eléctrica gestada con el Proyecto Mesoamérica (PM) a través del Sistema de Interconexión Eléctrica de los Países de América Central (Siepac), que plantea el desarrollo de 
energías renovables para abastecer la demanda industrial (Anderson, Pringle y Rojas, 2006; Delgado, 2010; Masís y Fernández, 2016).

En ese sentido Costa Rica promete ser uno de los más productivos, con una red hidrográfica de cerca de 110000 millones de $\mathrm{m}^{3}$ (Valverde, 2015). Esto ha generado que hayan empezado a salir a la luz una serie de dudas e irregularidades con respecto a la legitimidad, rigurosidad y eficacia de los Estudios de Impacto Ambiental (EsIA) para medir los efectos de este tipo de desarrollo sobre la vida silvestre y los ecosistemas (Anderson et al. 2006).

Por lo tanto, este trabajo tiene como objetivo conocer las especies de mamíferos no voladores que habitan en la cuenca del río Unión, así como comparar estos resultados con los del EsIA, realizado como requisito ante la Secretaría Técnica Nacional Ambiental (Setena) para la construcción del Proyecto Hidroeléctrico San Rafael (PH San Rafael) en el sureste del país.

\section{Materiales y métodos}

El río Unión (San Pedro de Pérez Zeledón, San José), coordenadas 9¹9'13'N, $83^{\circ} 31^{\prime} 01^{\prime \prime} \mathrm{W}$, se encuentra en una región húmeda tropical. Tiene una longitud aproximada de $12 \mathrm{~km}$, iniciando en la confluencia entre el río San Rafael y la quebrada Pavón a 867 msnm, en la localidad de San Rafael, hasta la confluencia con el río General, en la localidad de Santa Ana a 540 msnm. Los suelos a la orilla del río Unión han sido fragmentados principalmente para pastoreo y plantaciones de café, y el río ha sido usado para riego de cultivos piñeros con grandes consecuencias ambientales (Hernández, 2008).

El PH San Rafael (7250 KWh) ha sido propuesto por la empresa H. Solís en el río San Rafael, junto a otros 14 proyectos privados en otros ríos del valle de El General. Según Miranda (2013), el área de influencia directa (AID) corresponde al área de la construcción, es decir, desde la toma de agua hasta la zona donde se devuelve el agua al río, además de la casa de máquinas y otras infraestructuras, mientras que el área de influencia indirecta (AII), abarca desde la infraestructura hasta la confluencia con un río que ayude a compensar los cambios de caudal, es decir, unos $12 \mathrm{~km}$ río abajo desde el área del proyecto.

Muestreo: Se realizaron recorridos desde junio de 2014 a junio de 2015 en 12 parcelas de $15 \times 500 \mathrm{~m}$ a lo largo de $12 \mathrm{~km}$, las cuales fueron establecidas con el software ArcMap versión 10.0, en diferentes coberturas vegetales. Se identificaron huellas y se realizaron avistamientos directos. Además, se incluyen especies avistadas por lugareños conocedores del área, las cuales están indicadas con “*”. Las reportadas por Miranda (2013) se señalan con “(M)”.

Los datos sobre alimentación fueron tomados de Ecobiosis (2015), mientras que los de estado de conservación de la Unión Internacional para la Conservación de la Naturaleza (IUCN 2015) y Ecobiosis (2015). Las especies en peligro de extinción o con poblaciones reducidas han sido declaradas en la Ley de Conservación de Vida Silvestre $N^{\circ} 7317$, la Ley Orgánica del Ambiente $\mathrm{N}^{\circ} 7554$ y el Decreto $\mathrm{N}^{\circ}$ 26435-MINAE. Las que se prohíbe su comercio han sido declaradas en Convención sobre el Comercio Internacional de Especies Amenazadas de Fauna y Flora Silvestre (CITES).

\section{Resultados y discusión}

Se muestran 29 especies de mamíferos no voladores (Tabla 1), clasificados en 16 familias. Cuatro especies están bajo alguna categoría de amenaza. Diez se encuentran incluidas dentro de alguno de los tres Apéndices de CITES. Tres poseen poblaciones reducidas o amenazadas, mientras que otras tres se encuentran en peligro de extinción. Una no posee datos suficientes para incluirla dentro de algún grado de amenaza, mientras que las demás no se consideran amenazadas.

Por otro lado, el EsIA reportó ocho especies (27,5\% menor) clasificadas en siete familias. Solo una de las especies (D. punctata) se encuentra en el Apéndice III de CITES. Las demás se categorizan como de preocupación menor. Para las especies que se verían afectadas potencialmente por el PH San Rafael, Miranda (2013) establece como medida de 
TABLA 1

Mamíferos no voladores de la cuenca del río Unión, Costa Rica

\begin{tabular}{|c|c|c|c|}
\hline Familia y especie & Nombre común & Conservación & Alimentación \\
\hline \multicolumn{4}{|l|}{ Bradypodidae } \\
\hline Bradypus variegatus Schinz, $1825^{*}$ & Perezoso & MP, II & $\mathrm{H}$ \\
\hline \multicolumn{4}{|l|}{ Canidae } \\
\hline Canis latrans Say, 1823* & Coyote & MP & $\mathrm{F}, \mathrm{C}, \mathrm{I}$ \\
\hline \multicolumn{4}{|l|}{ Cervidae } \\
\hline Odocoileus virginianus (Zimmermann, 1780) & Venado & MP & $\mathrm{H}, \mathrm{F}, \mathrm{G} / \mathrm{S}$ \\
\hline \multicolumn{4}{|l|}{ Cuniculidae } \\
\hline Cuniculus paca (Linnaeus, 1766) & Tepezcuintle & MP, III & $\mathrm{F}, \mathrm{H}, \mathrm{G} / \mathrm{S}, \mathrm{I}$ \\
\hline \multicolumn{4}{|l|}{ Dasypodidae } \\
\hline Cabassous centralis (Miller, 1899)* & Armadillo zopilote & DI, III, PAR & I \\
\hline Dasypus novemcinctus Linnaeus, 1758 (M) & Cusuco & MP & $\mathrm{I}, \mathrm{F}, \mathrm{C}$ \\
\hline \multicolumn{4}{|l|}{ Dasyproctidae } \\
\hline Dasyprocta punctata Gray, 1842 (M) & Guatuza, cherenga & MP, III & $\mathrm{F}, \mathrm{H}, \mathrm{G} / \mathrm{S}$ \\
\hline \multicolumn{4}{|l|}{ Didelphidae } \\
\hline Philander oposum (Linnaeus, 1758) & Zorro cuatro ojos & MP & $\mathrm{F}, \mathrm{I}, \mathrm{C}$ \\
\hline Caluromys derbianus Waterhouse, 1841 & Zorro de balsa & MP & $\mathrm{F}, \mathrm{I}, \mathrm{N}$ \\
\hline Didelphis marsupialis Linnaeus, 1758 (M) & Zorro pelón & MP & $\mathrm{H}, \mathrm{C}, \mathrm{F}, \mathrm{I}, \mathrm{G} / \mathrm{S}$ \\
\hline Didelphis virginiana Kerr, 1792 & Zorro pecho amarillo & MP & $\mathrm{H}, \mathrm{C}, \mathrm{F}, \mathrm{I}, \mathrm{G} / \mathrm{S}$ \\
\hline Marmosa zeledoni (J.A. Allen, 1900)* (M) & Zorricí & MP & $F_{1} I$ \\
\hline \multicolumn{4}{|l|}{ Erethizontidae } \\
\hline Sphiggurus mexicanus (Kerr, 1792) (M) & Puercoespín & MP & $\mathrm{H}, \mathrm{F}, \mathrm{G} / \mathrm{S}$ \\
\hline \multicolumn{4}{|l|}{ Felidae } \\
\hline Herpailurus yagouaroundi (É. Geoffroy Saint- Hilaire, 1803)* & León breñero & $M P, I, P E$ & $\mathrm{C}$ \\
\hline Leopardus pardalis (Linnaeus, 1758)* & Manigordo, ocelote & $M P, I, P E$ & C \\
\hline Leopardus wiedii (Schinz, 1821) & Caucel, tigrillo & $C A, I, P E$ & $\mathrm{C}$ \\
\hline \multicolumn{4}{|l|}{ Geomyidae } \\
\hline Orthogeomys cavator (Bangs, 1902) & Taltuza & MP & $\mathrm{F}$ \\
\hline \multicolumn{4}{|l|}{ Leporidae } \\
\hline Silvilagus brasiliensis (Linnaeus, 1758) (M) & Conejo & MP & $\mathrm{H}$ \\
\hline \multicolumn{4}{|l|}{ Mephitidae } \\
\hline Conepatus semistriatus (Boddaert, 1785) & Zorro hediondo & MP & $\mathrm{I}, \mathrm{F}, \mathrm{C}$ \\
\hline \multicolumn{4}{|l|}{ Mustelidae } \\
\hline Eira barbara (Linnaeus, 1758) & Tolomuco & MP & $C, F, I, N$ \\
\hline Galictis vittata (Schreber, 1776) & Grisón, tejón & MP, III, PAR & $\mathrm{C}$ \\
\hline Lontra longicaudis (Olfers, 1818) & Nutria & CA, I, PAR & $\mathrm{P}, \mathrm{C}$ \\
\hline Mustela frenata Lichtenstein, 1831 & Comadreja & MP & $\mathrm{C}$ \\
\hline \multicolumn{4}{|l|}{ Myrmecophagidae } \\
\hline Tamandua mexicana (Saussure, 1860) & Oso hormiguero & MP & I \\
\hline \multicolumn{4}{|l|}{ Procyonidae } \\
\hline Nasua narica (Linnaeus 1766) & Pizote & MP & $C, F, I$ \\
\hline Potos flavus (Schreber, 1774) & Martilla & MP, III & $\mathrm{F}, \mathrm{I}, \mathrm{N}, \mathrm{C}$ \\
\hline Procyon lotor (Linnaeus, 1758) (M) & Mapache & MP & $\mathrm{C}, \mathrm{F}, \mathrm{I}, \mathrm{H}, \mathrm{P}, \mathrm{G} / \mathrm{S}$ \\
\hline \multicolumn{4}{|l|}{ Sciuridae } \\
\hline Sciurus granatensis Humboldt, 1811 (M) & Ardilla & MP & $\mathrm{F}, \mathrm{I}, \mathrm{G} / \mathrm{S}$ \\
\hline Sciurus variegatoides Ogilby 1839 & Ardilla & MP & $\mathrm{I}, \mathrm{F}, \mathrm{G} / \mathrm{S}$ \\
\hline
\end{tabular}

Fuente: Elaboración propia. Las especies con "M" son las reportadas por el EsIA coordinado por Miranda (2013), y con"*" las reportadas por los baqueanos. 


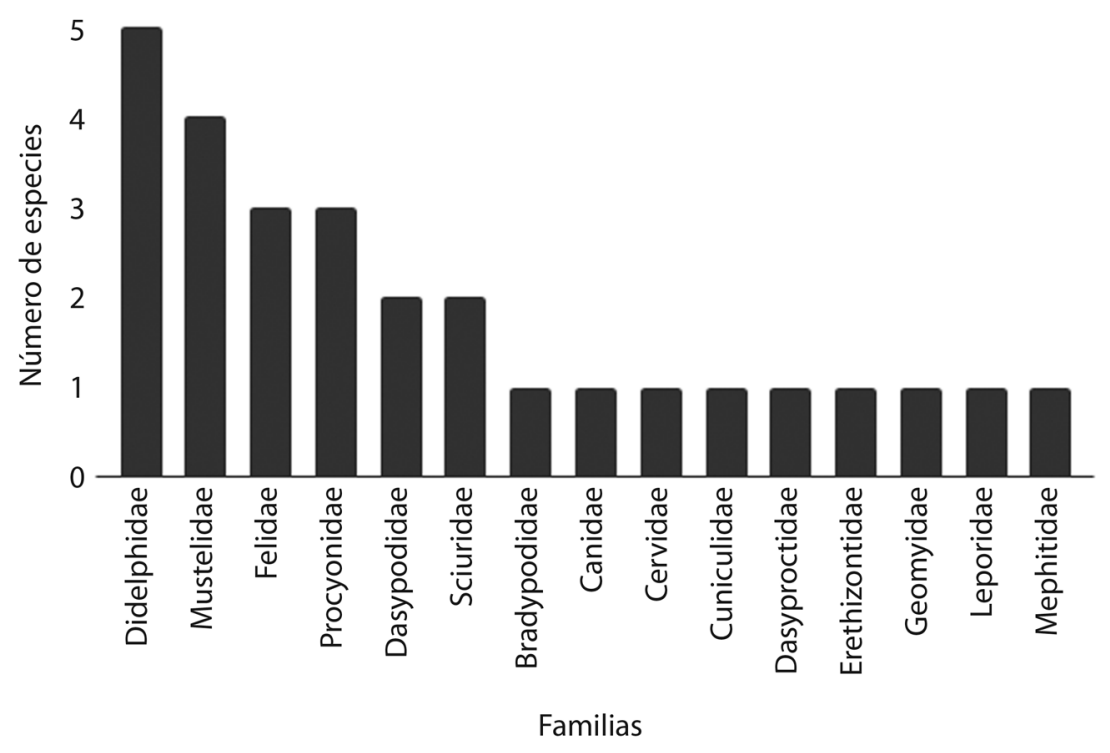

Figura 1. Representatividad de las familias de mamíferos de la cuenca del río Unión, Costa Rica.

compensación la restauración ecológica de las partes altas de la cuenca.

En la siguiente figura se muestra el número de especies por familias presentes del río Unión.

Con respecto a la Figura 1, la familia con más especies fue Didelphidae, al igual que en el estudio de Miranda (2013). Estos marsupiales cuentan con 92 especies, siete en Costa Rica. Son terrestres o arborícolas y habitan gran parte de los ecosistemas neotropicales (Wainwright, 2002). Mustelidae, con cuatro especies, fue la segunda más representada. Se compone de 65 especies, siete de ellas representadas en Costa Rica (Wainwright, 2002). Se caracterizan por ser terrestres o acuáticas, con hábitos alimenticios carnívoros, piscívoros o carroñeros.

En la siguiente figura se presentan hábitos alimenticios de las especies reportadas en el río Unión.

Para el caso de la Figura 2, los hábitos frugívoros, insectívoros y carnívoros son los más habituales, siendo la frugivoría la que más especies incluye. Algunos vegetales como Ficus spp (Moraceae), Anacardium excelsum (Anacardiaceae), Brosimum alicastrum (Moraceae), y otras, representan una importante fuente alimenticia (observación personal). Las especies carnívoras generalmente se alimentan de otros mamíferos (Ecobiosis, 2015).

Los piscívoros, nectarívoros y necrófagos solo estuvieron representados por dos, dos y una especie, respectivamente. Para el primer caso, el bienestar de las especies acuáticas es fundamental para su conservación (Spinola, 1994), mientras que para el segundo caso, al parecer la principal especie vegetal que brinda néctar a mamíferos es Ochroma pyramidale (Malvaceae) (Ecobiosis, 2015).

En el EsIA los principales hábitos alimenticios fueron frugívoro (cinco), herbívoro (cuatro), insectívoro y granívoro/semillero (tres), y carnívoro (dos). Algunas especies presentaron varios hábitos (Miranda, 2013). Al respecto resulta importante destacar el caso de la nutria, no citada en el EsIA, y con la cual se puede asimilar su vulnerabilidad a partir de estudios de impactos ictiológicos de los proyectos hidroeléctricos como el de Farah (2016), quien destaca impactos en la fragmentación ecosistémica y la afectación potencial para unas 90 especies de peces en el país, debido en gran parte por el caudal ecológico de $10 \%$ (90\% del caudal para la generación hidroeléctrica).

Una de las zonas protegidas más cercana que cuenta con estudios es el Corredor Biológico 


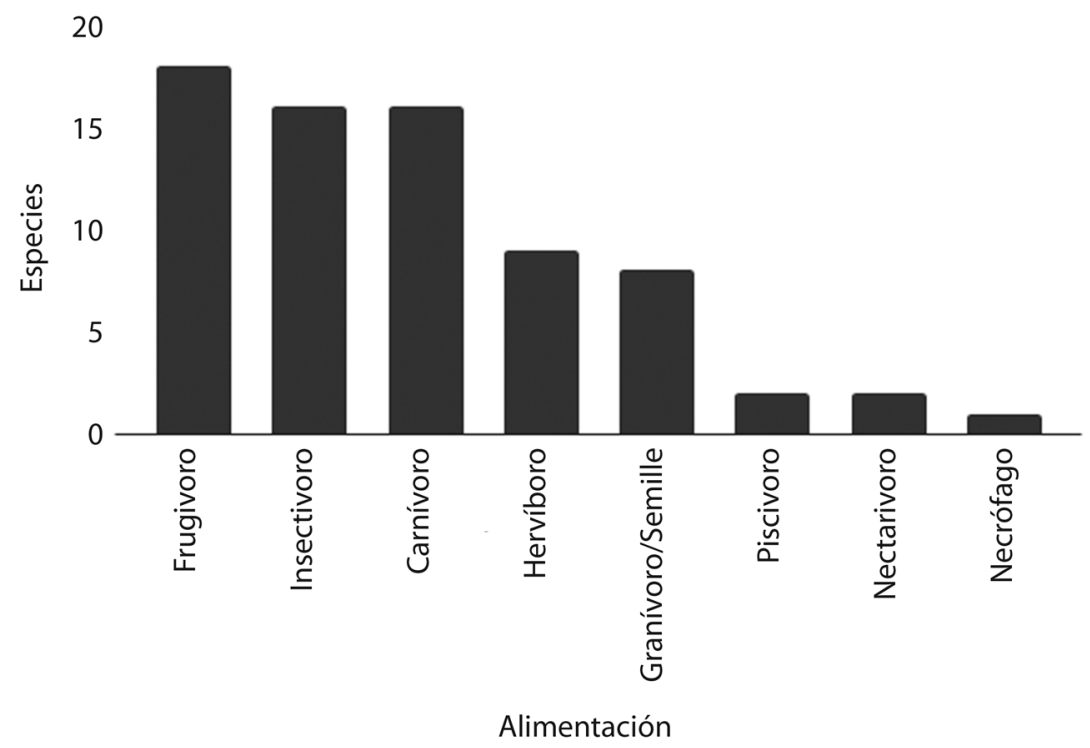

Figura 2. Número de especies por cada gremio alimenticio de los mamíferos de la cuenca del río Unión, Costa Rica.

Alexander Skutch, donde se han reportado unas 133 especies en cerca de 6000 ha; más del $50 \%$ de las especies conocidas en el país (Canet, 2005). La explicación de menos especies en el río Unión (aparte del área), radica en las pocas -a veces nulas- coberturas vegetales a lo largo de la cuenca, y en consecuencia, menor disponibilidad de alimento y hábitat. A pesar de eso, el EsIA no consideró los estudios biológicos realizados en zonas cercanas, y solo utilizó las guías clásicas de especies para la identificación a nivel nacional.

Las situaciones en torno a los impactos no conocidos de los proyectos hidroeléctricos ocurren por la poca información existente y las escasas discusiones acerca de los impactos biológicos de estos. Desde las ciencias sociales este tema ha sido más abordado desde la perspectiva de despojo hídrico (Masís y Fernández, 2016), pero desde las ciencias biológicas es una discusión incipiente que en sus primeros pasos ya ha brindado importantes aportes a escala nacional como el de Farah (2016) y algunos otros importantes trabajos previos en la vertiente del Caribe.

A esto se suma la situación de los grupos biológicos poco conocidos o complejos, los cuales, como lo menciona Miranda (2013) para el caso de los invertebrados acuáticos, no son evaluados por esas razones. Sin embargo, este no es el caso de los mamíferos. Al ser los EsIA financiados por la empresa desarrolladora, en parte por razones económicas las evaluciones no son rigurosas, y poseen, como en el caso de Miranda (2013), visitas de campo, al parecer insuficientes, así como de revisión de literatura que dependiendo de la zona, no es suficiente.

Las iniciativas hidroeléctricas, al responder a un plan geopolítico a nivel mesoamericano, deben agilizar su tramitología burocrática, tal como lo recomienda el Banco Mundial (2009), en una serie de pautas solicitadas al país en esa dirección. Esto es, evaluciones ambientales institucionales cada vez menos rigurosas con el fin no solo de agilizar los procesos, sino también de reducir los costos de producción, lo cual repercute negativamente tanto en la calidad-veracidad de los resultados, como en la omisión de especies y ecosistemas de importancia para la conservación que en este caso no son tomados en cuenta, lo cual puede leerse también una forma de evitar posibles trabas con la vida silvestre vulnerable o en peligro.

\section{Conclusiones}

A pesar de no haber utilizado métodos como cámaras trampa y redes, el número de especies aportadas es mayor a las reportadas 
por el EsIA (8 vs. 29) debido a que su estudio se basó solamente en el AID (toma de agua, casa de máquinas y embalse), y no en el AII. Además, a pesar de asegurar que tomó en cuenta reportes locales, esto no se ve reflejado en especies muy comunes -o que su presencia es conocida- que no fueron incluidas. Por lo tanto, las dos principales carencias del EsIA son el número de especies reportadas, y la omisión de especies comunes y/o sumamente importantes para la conservación.

El número de especies reportadas, aunque significativamente menor a zonas protegidas cercanas como Los Cusingos, devela la importancia de esta cuenca como corredor biológico y hábitat para estas especies de mamíferos, algunas de ellas declaradas vulnerables o en peligro. Estas especies habitan esta cuenca a pesar de la desaparición de los bosques ribereños en algunos sectores.

Los engranajes burocráticos y las presiones a nivel país respondiendo a los intereses geopolíticos regionales del PM, han facilitado la fiscalización superficial de los EsIA, llevando a que se conviertan en estudios poco rigurosos en aspectos biológicos. Por lo que la conservación de la naturaleza se vuelve un factor meramente político que se pliega de manera incluso antiecológica ante los intereses económicos.

La presentación de menor número de especies no solo se debe a un factor de rigurosidad, sino también de subestimación de la biodiversidad, lo cual podría facilitar los procesos y evitar trabas al no considerar especies de importancia nacional e internacional para la conservación, como el significativo caso de la especie $L$. longicaudis, protegida internacionalmente por diversas razones, pero no presentada en el EsIA.

\section{Recomendaciones}

Es necesario considerar las especies que no habían sido incluidas en el EsIA, para así evaluar los efectos en caso de construcción de este proyecto hidroeléctrico sobre todo para las especies vulnerables o en peligro, así como para las que dependen cercanamente del bienestar de los ecosistemas ribereños y del agua en sí misma.

Se recomienda entablar de forma prioritaria la discusión sobre los impactos biológicos de los proyectos hidroeléctricos y la necesidad de replantear mecanismos metodológicos, lo cual se vuelve urgente sobre todo en un contexto de proliferación de este tipo de energía que ya ha venido evidenciando sus problemáticas socioambientales.

Los estudios sobre afectaciones biológicas de los proyectos hidroeléctricos en Costa Rica se han centrado principalmente sobre la ictiofauna como grupo más afectado. Sin embargo, también se considera importante analizar la incidencia de estos proyectos sobre algunos mamíferos que pueden dar resultados relevantes, ya sea por su hábitat cercano a los cuerpos a agua, o por sus hábitos alimenticios principalmente ícticos, como el caso de L. longicaudis.

\section{Agradecimientos}

A Oscar Arias Segura, Carmen Campos Valverde, Ananias Ortiz, Federico Ortiz y Omar Arias por sus reportes de mamíferos.

\section{Nomenclatura}

Conservación: MP: Preocupación menor. DI: Datos insuficientes. CA: Casi amenazada. I, II o III: Apéndice CITES. PAR: Poblaciones amenazadas o reducidas (MINAE). PE: Peligro de extinción (MINAE).

Alimentación: I: Insectívoro. F: Frugívoro. G/S: Granívoro/Semillero. P: Piscívoro. C: Carnívoro. N: Nectarívoro. H: Herbívoro.

\section{Referencias}

Anderson, E., C. Pringle y M. Rojas. (2006). Transforming tropical rivers: an environmental perspective on hydropower development in Costa Rica. Aquatic Conserv: Mar. Freshw. Ecosyst. 16: 679-693.

Banco Mundial. (2009). Competitividad de Costa Rica. San José, Costa Rica. Banco Mundial. 36 pp.

Canet, D. (2005). Ficha técnica para el diseño y oficialización del Corredor Biológico Alexander Skutch. San 
José, Costa Rica. Centro Científico Tropical-Red de Pequeñas Reservas.

Carrillo, E. y C. Vaughan. (1993). Variación en el comportamiento de Procyon spp. (Carnivora: Procyonidae) por la presencia de turistas en un área silvestre de Costa Rica. Rev. Biol. Trop. 41(3): 843-848.

Delgado, G. (2010). Cambio de paradigma energético y las nuevas tecnologías. Temas de Nuestra América 26(49): 29-48.

Ecobiosis. (2015). Portal Nacional de Biodiversidad Costarricense. Recuperado de: http://www.ecobiosis. museocostarica.go.cr/

Farah, A. (2016). Fragmentación del hábitat por represas hidroeléctricas para la ictiofauna dulceacuícola en Costa Rica. San José, Costa Rica. Tesis de Licenciatura en Ecología y Gestión de Ambientes Acuáticos, Universidad de Costa Rica.

Hernández, C. (2008). Colonización agrícola e impacto ambiental en la cuenca del río General (1914-2004). Revista de Ciencias Ambientales 36(2): 29-33.

Hilje, L. y J. Monge. (1988). Diagnóstico preliminar acerca de los vertebrados que son plagas en Costa Rica. Manejo Integrado de Plagas 10: 39-52.

International Union for Conservation of Natural Resources (IUCN). (2015). The IUCN Red List of Threatened Species. Recuperado de: http://www.iucnredlist.org/

Masís, M.J y D. Fernández. (Coords.). (2016). Defensa de los ríos Convento y Sonador. La historia de nuestra lucha. San José, Costa Rica. Vicerrectoría de Acción Social-UCR.
Miranda, E. (2013). Proyecto Hidroeléctrico San Rafael. Estudio de Impacto Ambiental. San José, Costa Rica. Gestión Ambiental de Proyectos (GAPRO)-Grupo H. Solís. 161 pp.

Monge, J. y J. Linares. (2010). Presencia del zorro de cuatro ojos (Philander opossum) en el cultivo de piña (Ananas comosus). Agronomía Mesoamericana 21(2): 343-347.

Monge, J. (1996). Vertebrate mortality in tropical highways: The Costa Rican case. Vida Silvestre Neotropical 5: 154-156.

Mora, J. (2000). Los mamíferos silvestres de Costa Rica. San Pedro, Costa Rica. EUNED. 220 pp.

Rodríguez, B., J. Ramírez, D. Villalobos y R. Sánchez. (2014). Actualización de la lista de especies de mamíferos vivientes de Costa Rica. Mastozoología Neotropical 21(2): 275-289.

Sponila, R. y C. Vaughan. (1994). Dieta de la nutria neotropical (Lontra longicaudis) en la Estación Biológica La Selva, Costa Rica. Vida Silvestre Neotropical 4(2): 125-132.

Spinola, R. (1994). Abundancia relativa y actividad de marcaje de la nutria neotropical (Lontra longicaudis). Vida Silvestre Neotropical 4(1): 38-45.

Valverde, R. (2015). Disponibilidad, distribución, calidad y perspectivas del agua en Costa Rica. Revista de Ciencias Ambientales 45: 5-12.

Wainwright, M. (2002). The Natural History of Costa Rican Mammals. Miami, Estados Unidos. Zona Tropical Publication. 384 pp. 